\title{
Secrecy in Prepare-and-Measure Clauser-Horne-Shimony-Holt Tests with a Qubit Bound
}

\author{
Erik Woodhead ${ }^{1, *}$ and Stefano Pironio ${ }^{2}$ \\ ${ }^{1}$ ICFO-Institut de Ciències Fotòniques, avinguda Carl Friedrich Gauss 3, 08860 Castelldefels, Barcelona, Spain \\ ${ }^{2}$ Laboratoire d'Information Quantique, CP 224, Université libre de Bruxelles (ULB), 1050 Bruxelles, Belgium
}

(Received 13 July 2015; published 6 October 2015)

\begin{abstract}
The security of device-independent (DI) quantum key distribution (QKD) protocols relies on the violation of Bell inequalities. As such, their security can be established based on minimal assumptions about the devices, but their implementation necessarily requires the distribution of entangled states. In a setting with fully trusted devices, any entanglement-based protocol is essentially equivalent to a corresponding prepare-and-measure protocol. This correspondence, however, is not generally valid in the DI setting unless one makes extra assumptions about the devices. Here we prove that a known tight lower bound on the min entropy in terms of the Clauser-Horne-Shimony-Holt Bell correlator, which has featured in a number of entanglement-based DI QKD security proofs, also holds in a prepare-and-measure setting, subject only to the assumption that the source is limited to a two-dimensional Hilbert space.
\end{abstract}

The security of quantum key distribution (QKD) rests on tradeoffs inherent to quantum physics, such as the impossibility of state cloning, the measurement-disturbance tradeoff, or the monogamy of entanglement. Similarly, the security of device-independent (DI) QKD [1-3], which can be established with minimal assumptions about the internal functioning of the devices, is based on a fundamental tradeoff between the violation of Bell inequalities and the unpredictability of quantum measurements. The simplest setting in which this tradeoff can be stated involves two separate parties, Alice and Bob, sharing two subsystems in an entangled state on which they perform, respectively, one of two measurements $x, y \in\{0,1\}$ yielding one of two outcomes $a, b \in\{0,1\}$. In this setting, the expectation value

$$
S=\sum_{a b x y}(-1)^{a+b+x y} P(a b \mid x y)
$$

of the Clauser-Horne-Shimony-Holt (CHSH) Bell correlator [4], where $P(a b \mid x y)$ denotes the joint probabilities for outcomes $a, b$ given measurements $x, y$, implies the fundamental lower bound

$$
H_{\min }(A \mid E) \geq 1-\log _{2}\left(1+\sqrt{2-S^{2} / 4}\right)
$$

on the min entropy $H_{\min }(A \mid E)$ of Alice's outcome conditioned on one of Alice's inputs (say, $x=0$ ) and the quantum side information $E$ of any potential adversary. This relation is tight and is attained with equality with the optimal attack described in Ref. [3].

Contrarily to other tradeoffs used in standard QKD, which assume some level of trust and characterization of the quantum systems, the bound (2) is device independent in the sense that it holds for any quantum state $\rho_{A B E}$ and measurement operators $\left\{M_{a \mid x}\right\}$ and $\left\{M_{b \mid y}\right\}$ characterizing
Alice's and Bob's devices. The relation (2) was first derived in the context of DI-randomness certification [5] and has since featured as an ingredient in a number of DI QKD security proofs $[6-8]$.

Since they are based on the violation of Bell inequalities, DI QKD protocols are entanglement-based (EB) protocols. Indeed, in the DI setting, entanglement is necessary to guarantee security with a minimal set of assumptions on the devices [9]. Implementations of traditional (non-DI) QKD protocols, such as BB84 [10], are, however, usually of the prepare-and-measure (PM) type. In a PM protocol, Alice uses a source to prepare certain states which are then transmitted through a quantum channel to Bob who performs measurements on them. PM schemes have the practical advantage that they do not require the manipulation of entanglement. For this same reason, however, they cannot be fully DI. Recent works have nevertheless considered the possibility of PM QKD schemes that are at least partially DI $[11,12]$.

In traditional QKD, a famous argument establishes an equivalence between the security of $\mathrm{PM}$ and $\mathrm{EB}$ protocols [13]. In the BB84 protocol, for instance, Alice could prepare the four BB84 states by preparing a $\Phi^{+}$Bell state $\left(|0\rangle_{A}|0\rangle_{A^{\prime}}+|1\rangle_{A}|1\rangle_{A^{\prime}}\right) / \sqrt{2}$ (in some Hilbert space $\mathcal{H}_{A} \otimes \mathcal{H}_{A^{\prime}}$ ) in her lab and measuring either in the computational $\left(\left\{|0\rangle_{A},|1\rangle_{A}\right\}\right)$ basis or in the Hadamard $\left(\left\{|+\rangle_{A},|-\rangle_{A}\right\}\right)$ basis in $\mathcal{H}_{A}$ and transmitting the projected state in $\mathcal{H}_{A^{\prime}}$ to Bob. Since the security can only be reduced if the $\Phi^{+}$state is replaced by a state $|\psi\rangle_{A B E}$ chosen by an adversary (Eve) and shared between Alice, Bob, and Eve (the situation considered in EB security proofs), a security proof of the EB version of the BB84 protocol automatically implies the security of the PM version.

In the DI setting one can similarly associate a corresponding PM scheme to any EB scheme. In particular, one 


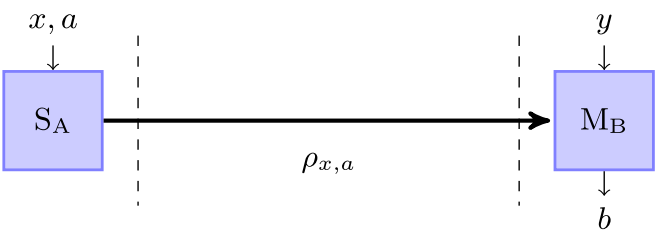

FIG. 1 (color online). Semi-device-independent scenario with the prepare-and-measure CHSH estimation. Alice's source $\left(S_{A}\right)$ can emit one of four different qubit states $\rho_{x, a} \in\left\{\rho, \rho^{\prime}, \sigma, \sigma^{\prime}\right\}$ depending on a choice of input $(x, a) \in\{0,1\}^{2}$. Bob's measurement device $\left(M_{B}\right)$ performs one of two measurements depending on a choice of input $y \in\{0,1\}$, yielding an outcome $b \in\{0,1\}$.

can consider a PM version of the above CHSH scenario, as illustrated in Fig. 1. In this PM version, Alice possesses a source which can emit one of four different quantum states, noted $\rho, \rho^{\prime}, \sigma$, and $\sigma^{\prime}$, depending on a respective choice of input $(x, a)=(0,0),(0,1),(1,0)$, and $(1,1)$. Alice randomly chooses $x \in\{0,1\}$ (not necessarily equiprobably) and chooses $a \in\{0,1\}$ randomly and equiprobably and attempts to transmit the corresponding state to Bob, who may perform one of two binary-outcome measurements on them (indexed by the input $y \in\{0,1\}$ and output $b \in\{0,1\})$. We can then define

$$
S=\frac{1}{2} \sum_{a b x y}(-1)^{a+b+x y} P(b \mid a x y)
$$

as the PM analogue of the CHSH correlator (1).

In a traditional (non-DI) setting, the equivalence between the EB and PM scenarios would imply that the bound (2) on Alice's randomness as a function of the CHSH correlator also holds in the PM version. In a DI setting, however, this equivalence is not immediate at all. First, the PM version cannot be fully DI (because the source could simply transmit Alice's choice of input classically). The security of a PM version will thus depend on some minimal assumption about the source. One possibility is to assume a dimension bound on one or more of the devices; such semi-DI PM schemes were proposed in Ref. [11]. Second, states prepared by measurements on half of an entangled pair satisfy a constraint called basis independence: if a set $\left\{\rho_{x}\right\}$ of states is prepared with associated probabilities $\left\{p_{x}\right\}$ by performing a measurement on half an entangled pair, the average state $\sum_{x} p_{x} \rho_{x}$ is independent of the measurement used to prepare it (a version of the nosignaling principle). The basis-independence constraint, however, need not be satisfied, and is actually explicitly relaxed, in the PM setting.

We show here that the fundamental bound on Alice's min entropy (2) nevertheless still holds in a semi-DI setting, with the PM version of the CHSH correlator (3) used in place of Eq. (1). Such a result can then be used to bring the semi-DI setting (for which security proofs are lacking) closer in line to known security results for DI QKD. In particular, the conditional min entropy can, for instance, be used to lower bound the Devetak-Winter key rate [14] in order to establish the security against collective attacks of a semi-DI QKD protocol based on the estimation of the CHSH correlator (3).

Dimension assumption. - Let us start by making precise the assumption that we need to derive Eq. (2) in the PM setting. During the transmission from Alice to Bob, an adversary may perform an arbitrary unitary operation on the states sent by Alice, with the intent of gaining some information about them. (More general quantum operations can be made unitary by enlarging the adversary's Hilbert space, according to Stinespring's dilation theorem.) Following this unitary attack, the emitted state $\rho_{x, a}$ is now shared between Bob and Eve [15], i.e., acts on a Hilbert space $\mathcal{H}_{B} \otimes \mathcal{H}_{E}$. We make the assumption that the two differences $\rho-\rho^{\prime}$ and $\sigma-\sigma^{\prime}$ between the source states (after the unitary attack) share their support on a common two-dimensional subspace $\mathcal{H}_{A}$ of $\mathcal{H}_{B} \otimes \mathcal{H}_{E}$. We refer to this condition as the qubit source assumption. We will later discuss the physical implications of this assumption; for now we simply take it as a mathematical condition satisfied by the states prepared by Alice's box.

A simple example illustrates the necessity of the qubit source assumption. Specifically, if Alice's source prepares pure states $\rho_{x, a}=\left|\psi_{x, a}\right\rangle\left\langle\psi_{x, a}\right|$, which are duplicate copies of the BB84 states,

$$
\begin{array}{ll}
\left|\psi_{00}\right\rangle=|0\rangle_{B}|0\rangle_{E}, \quad\left|\psi_{01}\right\rangle=|1\rangle_{B}|1\rangle_{E}, \\
\left|\psi_{10}\right\rangle=|+\rangle_{B}|+\rangle_{E}, \quad\left|\psi_{11}\right\rangle=|-\rangle_{B}|-\rangle_{E},
\end{array}
$$

in which $|0\rangle$ and $|1\rangle$ are orthonormal and $| \pm\rangle=$ $\frac{1}{\sqrt{2}}[|0\rangle \pm|1\rangle]$, the maximal value $S=2 \sqrt{2}$ can be attained while Eve always acquires exactly the same state as Bob. These states are not linearly independent (one can readily verify that $|0\rangle|0\rangle+|1\rangle|1\rangle=|+\rangle|+\rangle+|-\rangle|-\rangle)$ and span a three-dimensional Hilbert space, from which we see that the security of the semi-DI scenario is fully compromised if the qubit source assumption is not satisfied.

Min entropy and Eve's distinguishability.-To prove the bound (2), let us first note that if the input $a$ is chosen equiprobably, its min entropy, conditioned on the case $x=0$ and on Eve's quantum side information, is a function of the classical-quantum state

$$
\tau_{A E}=\frac{1}{2}|0\rangle\left\langle 0\left|\otimes \rho_{E}+\frac{1}{2}\right| 1\right\rangle\langle 1| \otimes \rho_{E}^{\prime},
$$

in which $\rho_{E}$ and $\rho_{E}^{\prime}$ are Eve's marginals of the states $\rho$ and $\rho^{\prime}$ after some given unitary attack (in the rest of this article, subscripts indicate partial tracing in the obvious way, e.g., $\rho_{B}=\operatorname{Tr}_{E}[\rho]$ ). Evaluated on Eq. (6), the min entropy can be expressed [16,17] as

$$
H_{\min }(A \mid E)=1-\log _{2}\left(1+D\left(\rho_{E}, \rho_{E}^{\prime}\right)\right),
$$


with the trace distance between $\rho_{E}$ and $\rho_{E}^{\prime}$ defined by $D\left(\rho_{E}, \rho_{E}^{\prime}\right)=\frac{1}{2}\left\|\rho_{E}-\rho_{E}^{\prime}\right\|_{1}$, where $\|A\|_{1}=\operatorname{Tr}\left[\sqrt{A^{\dagger} A}\right]$ denotes the trace norm of an operator $A$. We will obtain the main result (2) by showing that the trace distance appearing in Eq. (7) is upper bounded by

$$
D\left(\rho_{E}, \rho_{E}^{\prime}\right) \leq \sqrt{2-S^{2} / 4}
$$

in terms of $S$.

Outline of proof.-We now outline the derivation of Eq. (8). The lengthier and more pedestrian parts of the proof are given in the Supplemental Material [18].

Let us first introduce operators $Z$ and $X$ defined such that

$$
\begin{aligned}
& \rho-\rho^{\prime}=\alpha Z, \\
& \sigma-\sigma^{\prime}=\beta X,
\end{aligned}
$$

with $\alpha=\frac{1}{2}\left\|\rho-\rho^{\prime}\right\|_{1}$ and $\beta=\frac{1}{2}\left\|\sigma-\sigma^{\prime}\right\|_{1}$ such that the (traceless qubit) operators $Z$ and $X$ satisfy $\frac{1}{2}\|Z\|_{1}=$ $\frac{1}{2}\|X\|_{1}=1$. Then, in terms of these operators, the CHSH expectation value (3) can be expressed as

$$
S=\frac{1}{2} \operatorname{Tr}\left[U_{B}\left(\alpha Z_{B}+\beta X_{B}\right)+V_{B}\left(\alpha Z_{B}-\beta X_{B}\right)\right],
$$

where $U_{B}=\sum_{b}(-1)^{b} M_{b \mid y=0}$ and $V_{B}=\sum_{b}(-1)^{b} M_{b \mid y=1}$ are Hermitian unitary operators acting on $\mathcal{H}_{B}$ describing the observables corresponding to the (without loss of generality, projective) measurements $y=0$ and $y=1$.

A general result for any pair of Hermitian unitaries is that they admit a common block diagonalization in blocks of dimension no more than 2 . We can thus set

$$
U_{B}=\bigoplus_{k} U_{B}^{k}, \quad V_{B}=\bigoplus_{k} V_{B}^{k},
$$

in which $U_{B}^{k}$ and $V_{B}^{k}$ are still Hermitian and unitary and of dimension at most 2, $\forall k$ (the Jordan lemma [19], see Lemma 2 of Ref. [9] for a short proof). This reduces the problem to considering qubit subspaces on Bob's side. For each subspace $k$, we can define the corresponding contribution to $S$ by

$S_{k}=\frac{1}{2} \alpha \operatorname{Tr}\left[\left(U_{B}^{k}+V_{B}^{k}\right) Z_{B}\right]+\frac{1}{2} \beta \operatorname{Tr}\left[\left(U_{B}^{k}-V_{B}^{k}\right) X_{B}\right]$,

with $\sum_{k} S_{k}=S$. Similarly, we define a probabilistic weight for each subspace $k$ by

$$
p_{k}=\frac{1}{2} \operatorname{Tr}\left[\mathbb{1}_{B}^{k} \mathcal{I}_{B}\right],
$$

with $\sum_{k} p_{k}=1$, defined in terms of the projection operator $\mathbb{1}_{B}^{k}$ on the $k$ th subspace (satisfying $\mathbb{1}_{B}^{k}=\left(U_{B}^{k}\right)^{2}=\left(V_{B}^{k}\right)^{2}$ ) and the partial trace $\mathcal{I}_{B}=\operatorname{Tr}_{E}[\mathcal{I}]$ of the identity on the space of source states (satisfying $\mathcal{I}=Z^{2}=X^{2}$ ).
We now introduce an orthonormal basis $\left\{|y\rangle,\left|y^{\prime}\right\rangle\right\}$ of the space of source states chosen such that $Y=|y\rangle\langle y|-$ $\left|y^{\prime}\right\rangle\left\langle y^{\prime}\right|$ is orthogonal to the operators $Z$ and $X$, defined by Eqs. (9) and (10), on the Bloch sphere. In this basis, in an appropriate phase convention, $Z$ and $X$ can be expressed as

$$
\begin{aligned}
& Z=e^{i(\varphi / 2)}|y\rangle\left\langle y^{\prime}\left|+e^{-i(\varphi / 2)}\right| y^{\prime}\right\rangle\langle y|, \\
& X=e^{-i(\varphi / 2)}|y\rangle\left\langle y^{\prime}\left|+e^{i(\varphi / 2)}\right| y^{\prime}\right\rangle\langle y|,
\end{aligned}
$$

for some (a priori unknown) angle $\varphi$, while the source space identity operator $\mathcal{I}$ takes the expression

$$
\mathcal{I}=|y\rangle\left\langle y|+| y^{\prime}\right\rangle\left\langle y^{\prime}\right| .
$$

One can readily verify that $\{Z, Y\}=\{X, Y\}=0$, that $[Z, X]=2 i \sin (\varphi) Y$, and that $\{Z, X\}=\cos (\varphi) \mathcal{I}$. An important step in the derivation of the trace-distance bound (8) consists in turning the value of $S$ into a constraint on the part $Y_{B}$ of the operator $Y$ accessible to Bob [20]. Specifically, in each subspace $k$ defined by the block diagonalization (12), we prove in Supplemental Material A [18] that there exists a Hermitian unitary operator $W_{B}^{k}$ with the property that

$$
\alpha \frac{1}{2} \operatorname{Tr}\left[W_{B}^{k} Y_{B}\right] \geq \sqrt{S_{k}^{2} / 4-p_{k}^{2}},
$$

where $S_{k}$ and $p_{k}$ are as defined in Eqs. (13) and (14). Equation (18) holds regardless of the value of $\beta$ appearing in Eq. (13) and of $\varphi$ in Eqs. (15) and (16). Note that $\alpha$ can also be eliminated using that $\frac{1}{2} \operatorname{Tr}\left[W_{B}^{k} Y_{B}\right] \geq \alpha \frac{1}{2} \operatorname{Tr}\left[W_{B}^{k} Y_{B}\right]$.

In order to obtain the upper bound (8) on $D\left(\rho_{E}, \rho_{E}^{\prime}\right)$, we also derive a tradeoff between the quantity $\frac{1}{2} \operatorname{Tr}\left[W_{B}^{k} Y_{B}\right]$, appearing in Eq. (18), and the distinguishability of Eve's states. Specifically, we prove in Supplemental Material B [18] that, for any Hermitian unitary $U_{E}$ acting on $\mathcal{H}_{E}$, the inequality

$$
\frac{1}{4} \operatorname{Tr}\left[W_{B}^{k} Y_{B}\right]^{2}+\frac{1}{4} \operatorname{Tr}\left[\left(\mathbb{1}_{B}^{k} \otimes U_{E}\right) Z\right]^{2} \leq p_{k}^{2}
$$

holds in each subspace $k$.

We obtain Eq. (8) by taking for $U_{E}$ in Eq. (19) a Hermitian unitary such that $\frac{1}{2} \operatorname{Tr}\left[U_{E} Z_{E}\right]=\frac{1}{2}\left\|Z_{E}\right\|_{1}$ [21]. Because $D\left(\rho_{E}, \rho_{E}^{\prime}\right)=\alpha \frac{1}{2}\left\|Z_{E}\right\|_{1} \leq \frac{1}{2}\left\|Z_{E}\right\|_{1}$, the trace distance is upper bounded by

$$
D\left(\rho_{E}, \rho_{E}^{\prime}\right) \leq \sum_{k} \frac{1}{2} \operatorname{Tr}\left[\left(\mathbb{1}_{B}^{k} \otimes U_{E}\right) Z\right] .
$$

Using Eqs. (18) and (19) and omitting $\alpha$, we have

$$
\frac{1}{2} \operatorname{Tr}\left[\left(\mathbb{1}_{B}^{k} \otimes U_{E}\right) Z\right] \leq p_{k} \sqrt{2-\left(S_{k} / p_{k}\right)^{2} / 4},
$$

and substituting Eq. (21) into Eq. (20) and using that the function $S \mapsto \sqrt{2-S^{2} / 4}$ is concave, we finally obtain 


$$
\begin{aligned}
D\left(\rho_{E}, \rho_{E}^{\prime}\right) & \leq \sum_{k} \frac{1}{2} \operatorname{Tr}\left[\left(\mathbb{1}_{B}^{k} \otimes U_{E}\right) Z\right] \\
& \leq \sum_{k} p_{k} \sqrt{2-\left(S_{k} / p_{k}\right)^{2} / 4} \\
& \leq \sqrt{2-\left(\sum_{k} S_{k}\right)^{2} / 4} \\
& =\sqrt{2-S^{2} / 4} .
\end{aligned}
$$

Combining with the expression (7) for the min entropy, we obtain Eq. (2).

As with its EB counterpart, Eq. (8) and the resulting min-entropy bound are tight and are attained with a PM version of the optimal attack originally given in Ref. [3]; for completeness we have included a description of this attack in Supplemental Material C [18].

Discussion of the qubit assumption.-Having proven our main result, let us now discuss the qubit source assumption in more detail. Note first that Alice's "preparation" device may not in general actually prepare a new state from scratch, but instead implement a transformation on a preexisting qubit stored in her box, which could be entangled with Eve's system prior to the protocol. The presence of such prior entanglement between Alice's device and Eve may completely break the security of a PM scheme, as noted in Ref. [11]. However, since our qubit assumption is formulated in the total space $\mathcal{H}_{B} \otimes \mathcal{H}_{E}$ including Eve's Hilbert space, it naturally limits the amount of potential prior entanglement between Alice and Eve (or Alice and Bob) and thus a nice mathematical feature of our formulation is that we do not need to state this limitation on prior entanglement as a separate, additional assumption.

On the other hand, since our qubit assumption is formulated in the space $\mathcal{H}_{B} \otimes \mathcal{H}_{E}$ after Eve's attack, it may not be possible to practically verify this assumption in a cryptographic setting (since Alice and Bob do not have access to Eve's system). Note, however, that a sufficient condition for our assumption to be satisfied is that (i) there exists no prior entanglement between Alice and Eve or Bob (e.g., Alice's preparation box has no quantum memory), and (ii) the states sent by Alice's box, before going through the channel and suffering Eve's (without loss of generality, unitary) attack, are such that $\rho-\rho^{\prime}$ and $\sigma-\sigma^{\prime}$ have support in the same two-dimensional subspace. Under these conditions, the states $\rho-\rho^{\prime}$ and $\sigma-\sigma^{\prime}$ after Eve's unitary attack will still share the same two-dimensional support and thus our qubit source assumption will be satisfied. However, the condition that we use to derive the minentropy bound (2) is formally weaker than the combination of (i) and (ii) as these only represent sufficient conditions for our assumption to be satisfied.

Another nice feature of our formulation is that the qubit assumption refers only to the differences $\rho-\rho^{\prime}$ and $\sigma-\sigma^{\prime}$ and not directly to the states $\rho_{x, a}$ themselves, which may live in a higher dimensional Hilbert space. For instance, in an optical implementation, each "qubit" may be a qubit encoded in the polarization degree of freedom of a single photon, but may also possess a vacuum component and thus formally be a three-level system of the form $\rho_{x, a}=p|0\rangle\langle 0|+(1-p) \tilde{\rho}_{a, x}$, where $\tilde{\rho}_{a, x}$ is the one-photon polarized qubit part. Still, the differences $\rho-\rho^{\prime}$ and $\sigma-\sigma^{\prime}$ only involve the genuine qubit parts and thus satisfy our qubit source assumption.

Finally, let us remark that our assumption can immediately be weakened in two ways. First, using convexity arguments, it is easy to see that the min-entropy bound (2) still holds if Alice's, Bob's, and Eve's systems share prior classical randomness, provided that for any value $\lambda$ of the shared randomness, the differences $\rho_{\lambda}-\rho_{\lambda}^{\prime}$ and $\sigma_{\lambda}-\sigma_{\lambda}^{\prime}$ satisfy the qubit assumption. Again, it may not be possible to practically verify this assumption in the most general DI setting (as Alice and Bob will not have access to the individual values of the shared randomness if their devices are uncharacterized). However, a sufficient condition for this assumption to be satisfied is if each of the averaged states $\rho_{x, a}=\sum_{\lambda} q_{\lambda} \rho_{x, a ; \lambda}$ are contained in the same qubit space, a condition which does not require any knowledge of the shared randomness.

Second, we point out that the bound on the min entropy is also robust with respect to the qubit assumption; i.e., this assumption need only be approximately verified. Specifically, suppose that, instead of assuming Eqs. (9) and (10), we assume that there exist traceless twodimensional unit operators $\alpha \tilde{Z}$ and $\beta \tilde{X}$ such that $\frac{1}{2}\left\|\left(\rho-\rho^{\prime}\right)-\alpha \tilde{Z}\right\|_{1} \leq \varepsilon$ and $\frac{1}{2}\left\|\left(\sigma-\sigma^{\prime}\right)-\beta \tilde{X}\right\|_{1} \leq \varepsilon$. Then it is easy to see that $D\left(\rho_{E}, \rho_{E}^{\prime}\right) \leq \frac{1}{2}\left\|\alpha \tilde{Z}_{E}\right\|_{1}+\varepsilon$ and that the CHSH expectation value computed with $\alpha \tilde{Z}$ and $\beta \tilde{X}$ cannot differ from $S$ by more than $4 \varepsilon$. Small deviations from the qubit source assumption can thus be tolerated, with a bound on the min entropy no worse than

$H_{\min }(A \mid E) \geq 1-\log _{2}\left(1+\sqrt{2-(S-4 \varepsilon)^{2} / 4}+\varepsilon\right)$.

Conclusion.-We gave a proof that the fundamental lower bound (2) on the randomness of Alice's outcomes as a function of the CHSH expression, originally derived in the context of device-independent QKD and randomness certification, still holds in a PM setting with a qubit assumption. Though the equivalence between EB and PM schemes in standard QKD may a priori suggest that this should naturally be the case, this is not at all immediate as this equivalence breaks in a DI setting. Indeed, the techniques that we have used here to establish the lower bound (2) in the PM setting are quite different from the ones used to establish the EB version of this bound.

This fundamental lower bound (2) can now, in principle, be used as a building block to prove the security of semi-DI 
QKD protocols, in the same way that it was used in the fully DI setting in Refs. [6-8].

We remark that in the EB scenario, an analogous tight bound for the Holevo quantity (or, equivalently, the conditional von Neumann entropy) instead of the min entropy had earlier been presented in Ref. [3] as part of a security proof against collective attacks. The conditional von Neumann entropy can likewise, in principle, be bounded in the PM scenario. A partial result for the von Neumann entropy, restricted to the case where Bob's measurements are additionally assumed to be two-dimensional, is given in Ref. [22].

Finally, having shown that our min-entropy bound holds for Alice's system conditioned on Eve, it would be interesting to investigate whether a similar result holds for the min-entropy $H_{\min }(B \mid E)$ associated with Bob's measurement outcome. In particular, a version of this result conditioned on just one of Alice's state preparations would apply immediately to the problem of randomness certification [5,23-25], which has similarly been investigated in PM scenarios [26,27].

This work is supported by the Spanish project FOQUS, the Generalitat de Catalunya (SGR 875), the EU projects QITBOX and QALGO, the F. R. S.-FNRS under the project DIQIP, and by the Interuniversity Attraction Poles program of the Belgian Science Policy Office under the Grant No. IAP P7-35 photonics@be. S.P. is a Research Associate of the Fonds de la Recherche Scientifique F. R.S.-FNRS (Belgium). E. W. was supported by a Belgian Fonds pour la Formation à la Recherche dans l'Industrie et dans l'Agriculture (F. R. I. A.) grant while this work was started.

*Erik.Woodhead@icfo.es

[1] A. K. Ekert, Phys. Rev. Lett. 67, 661 (1991).

[2] D. Mayers and A. Yao, in Proceedings of the 39th Annual Symposium on Foundations of Computer Science (IEEE Computer Society, Los Alamitos, 1998), p. 503.

[3] A. Acín, N. Brunner, N. Gisin, S. Massar, S. Pironio, and V. Scarani, Phys. Rev. Lett. 98, 230501 (2007).

[4] J. F. Clauser, M. A. Horne, A. Shimony, and R. A. Holt, Phys. Rev. Lett. 23, 880 (1969).

[5] S. Pironio, A. Acín, S. Massar, A. Boyer de La Giroday, D. N. Matsukevich, P. Maunz, S. Olmschenk, D. Hayes, L. Luo, T. A. Manning, and C. Monroe, Nature (London) 464, 1021 (2010).

[6] L. Masanes, S. Pironio, and A. Acín, Nat. Commun. 2, 238 (2011).

[7] S. Pironio, L. Masanes, A. Leverrier, and A. Acín, Phys. Rev. X 3, 031007 (2013).
[8] U. Vazirani and T. Vidick, Phys. Rev. Lett. 113, 140501 (2014).

[9] S. Pironio, A. Acín, N. Brunner, N. Gisin, S. Massar, and V. Scarani, New J. Phys. 11, 045021 (2009).

[10] C. H. Bennett and G. Brassard, in Proceedings of The IEEE International Conference on Computers, Systems and Signal Processing (IEEE, New York, 1984), p. 175.

[11] M. Pawłowski and N. Brunner, Phys. Rev. A 84, 010302(R) (2011).

[12] M. F. Pusey, J. Opt. Soc. Am. B 32, A56 (2015).

[13] C. H. Bennett, G. Brassard, and N. D. Mermin, Phys. Rev. Lett. 68, 557 (1992).

[14] I. Devetak and A. Winter, Proc. R. Soc. A 461, 207 (2005).

[15] In an abuse of notation, we will write $\rho_{x, a}$ to refer both to the states leaving Alice's device and to the states after Eve's unitary attack.

[16] R. König, R. Renner, and C. Schaffner, IEEE Trans. Inf. Theory 55, 4337 (2009).

[17] E. Woodhead, C. C. W. Lim, and S. Pironio, Theory of Quantum Computation, Communication, and Cryptography, Lecture Notes in Computer Science (Springer, Berlin, Heidelberg, 2013), Vol. 7582, p. 107.

[18] See Supplemental Material at http://link.aps.org/ supplemental/10.1103/PhysRevLett.115.150501 for proofs of intermediate results and the description of an optimizing attack.

[19] C. Jordan, Bull. Soc. Math. Fr. 3, 103 (1875).

[20] Roughly, if we think of Alice's states as living on the $\sigma_{z}-\sigma_{x}$ plane, we want to show that an attack that doesn't degrade $S$ too much also can't affect the $\sigma_{y}$ basis too much.

[21] Such a unitary can be constructed explicitly by diagonalizing $Z_{E}$. Specifically, if $Z_{E}=\sum_{k} \lambda_{k}|k\rangle\langle k|$ is an expression for $Z_{E}$ in diagonal form, then, for instance, $U_{E}$ can be taken to be $U_{E}=\sum_{k \in K_{\geq}}|k\rangle\left\langle k\left|-\sum_{k \in K_{<}}\right| k\right\rangle\langle k|$, where $K_{\geq}=\left\{k: \lambda_{k} \geq 0\right\}$ and $K_{<}=\left\{k: \lambda_{k}<0\right\}$ are the sets of indices corresponding, respectively, to the non-negative and negative eigenvalues of $Z_{E}$.

[22] E. Woodhead, Ph. D. thesis, Université libre de Bruxelles, 2014.

[23] R. Colbeck, Ph. D. thesis, University of Cambridge, 2006, arXiv:0911.3814.

[24] U. Vazirani and T. Vidick, in Proceedings of the FortyFourth Annual ACM Symposium on Theory of Computing, STOC '12 (ACM, New York, NY, 2012), p. 61.

[25] C. A. Miller and Y. Shi, in Proceedings of the 46th Annual ACM Symposium on Theory of Computing, STOC '14 (ACM, New York, NY, 2014), p. 417.

[26] H.-W. Li, Z.-Q. Yin, Y.-C. Wu, X.-B. Zou, S. Wang, W. Chen, G.-C. Guo, and Z.-F. Han, Phys. Rev. A 84, 034301 (2011).

[27] T. Lunghi, J. B. Brask, C. C. W. Lim, Q. Lavigne, J. Bowles, A. Martin, H. Zbinden, and N. Brunner, Phys. Rev. Lett. 114, 150501 (2015). 